\title{
$\beta$-adrenergic Receptor Activity in the Hippocampal Dentate Gyrus Participates in Spatial Learning and Memory Impairment in Sleep-deprived Rats
}

\author{
Huan-Jun $\mathrm{Lu}^{1}$ and Jing $\mathrm{lv}^{2 *}$ \\ ${ }^{1}$ Institute of Pain Medicine and Special Environmental Medicine, Nantong University, Jiangsu 226019, \\ ${ }^{2}$ Department of Physiology, Hebei University of Engineering, Hebei 056000, China
}

\begin{abstract}
Sleep deprivation (SD) leads to cognitive impairment, especially hippocampus-dependent learning and memory (L\&M). The hippocampal dentate gyrus (DG) is the key structure involved in spatial L\&M while long-term potentiation (LTP) is an important cellular mechanism responsible for L\&M. Physiological and behavioral evidences support the hypothesis that norepinephrine (NE) and $\beta$-adrenoceptors ( $\beta$-AR) may play an important role in regulating L\&M, including LTP. However, it is enigmatic how $\beta$-AR influences the LTP disruption or memory impairment under SD circumstances. In the present study, the rats were subjected to SD for $18 \mathrm{~h}$ per day for 21 consecutive days and cognitive capacity was assessed by the Morris water maze (MWM) test. We examined the extracellular concentration of NE in the DG using in vivo brain microdialysis and HPLC analysis. The amplitudes of field excitatory postsynaptic potential (fEPSP) were subsequently measured in the DG during MWM test in freely moving conscious rats. The extracellular concentrations of NE and fEPSP amplitudes in the DG were significantly increased during MWM test, while these responses were suppressed in SD rats. When fEPSP amplitudes in the DG were measured after local injection of isoproterenol (an agonist of $\beta$-AR), SD rats significantly alleviated the fEPSP impairment and rescued deficits of spatial L\&M. In addition, the reduced expression of N-methyl-Daspartate (NMDA) and $\alpha$-amino-3-hydroxy-5-methylisoxazole-4-propionic acid (AMPA) receptors in SD rats significantly increased by activation of $\beta$-AR by isoproterenol in the DG. In conclusion, we propose that $\beta$-adrenergic signaling can improve memory impairment in sleep-deficient rats by regulating synaptic efficiency and glutamatergic receptor expression.
\end{abstract}

Key words: : $\beta$-adrenoceptors, Hippocampal dentate gyrus, Sleep deprivation, Long-term potentiation, Spatial learning and memory

\section{INTRODUCTION}

It is widely acknowledged that sleep is crucial for proper brain function. Poor sleep, a common phenomenon in daily life, has been recognized as a risk factor for multiple disease states such as psychiatric disorders [1] and leads to severe cognitive impairment, including decline of attention, decision making and memory impairments [2-5]. The hippocampus is a key structure of learning

Submitted December 10, 2020, Revised January 26, 2021,

Accepted February 23, 2021

* To whom correspondence should be addressed.

TEL: 86-15530025752

e-mail:lvjing@hebeu.edu.cn and memory (L\&M) processes, and many reports have shown that sleep deprivation (SD) causes hippocampus-dependent memory impairment. For example, SD for $48 \mathrm{~h}$ degrades hippocampusdependent spatial L\&M performance in rats [5]. Likewise, a brief $5 \sim 6 \mathrm{~h}$ period of SD has been shown to impair the consolidation of object-place memory for which the hippocampus is required [6]. Long-term potentiation (LTP), a long-lasting change in the strength of synaptic connections, is a widely studied cellular model of synaptic plasticity and is believed to be responsible for the hippocampus-dependent L\&M [7, 8]. Rapid-eye movement (REM) sleep restriction for 21 days decreased LTP and long-term memory, and even 3 4 hours of total SD already reduced LTP in the granule cells of the dentate gyrus (DG) $[9,10]$. These results
Copyright (c) Experimental Neurobiology 2021. www.enjournal.org
This is an Open Access article distributed under the terms of the Creative Commons Attribution Non-Commercial License (http://creativecommons.org/licenses/by-nc/4.o) which permits unrestricted non-commercial use, distribution, and reproduction in any medium, provided the original work is properly cited. 
suggest that the hippocampus is especially vulnerable to SD.

The mammalian hippocampus is mainly divided into the CA1, CA3 and DG, and each subregion serves different functions in L\&M processes. The DG is defined as the functional gateway in the hippocampus due to the filtering role of synaptic information [11]. In addition, a variety of physiological evidences and computational modeling suggest that the DG plays a critical role not only in L\&M but also in spatial coding because the formation of spatial representation is damaged in rats with DG lesions [1214]. Although numerous studies are showing that SD worsens the function of the DG, they mainly focus on granular cell dependent adult neurogenesis [12]. However, underlying neurobiological mechanisms remain to be determined.

Norepinephrine (NE) is one of the important neurotransmitter essential for the induction and the maintenance of LTP that is extensively distributed in the central nervous system [15]. Noradrenergic fibers originate mainly in the locus coeruleus (LC), and their projection is widely connected throughout the forebrain, especially in the hippocampus [16]. More importantly, noradrenergic projection is enriched and the $\beta$-adrenergic receptor $(\beta$-AR) is highly expressed in the DG $[16,17]$. One behavioral study suggests that activation of $\beta$-AR by NE is required for the spatial learning and retrieval of associative memories [18]. $\beta$-adrenergic signaling in the DG also modulates learning-dependent LTP (LD-LTP) [19]. Moreover, inhibition of $\beta$-AR in the DG prevents spatial learning and disables the persistence of LTP [20].

Therefore, we hypothesized that NE transmission via $\beta$-AR may regulate $\mathrm{L} \& \mathrm{M}$ in SD model. We found that pharmacological activation of $\beta$-AR alleviated decreased fEPSP and memory deficits in conscious SD rats, and the expression of glutamatergic receptors were recovered by $\beta$-AR activation. Hence, this study aims at explaining the molecular mechanism of $\beta$-adrenergic signaling in sleep-deprived memory impairment.

\section{MATERIALS AND METHODS}

\section{Animals}

Male Sprague-Dawley rats (250 300 g) were obtained from Beijing Vital River Laboratory Animal Technology Co. Ltd. All protocols were approved by the Institutional Animal Care and were in accordance with the National Institutes of Health Guide for the Care and Use of Laboratory Animals. All efforts were made to minimize animal suffering and to reduce the number of animals used.

The animals were divided randomly into the following groups: (a) control and SD groups: the extracellular concentrations of NE and amplitudes of field excitatory postsynaptic potential (fEPSP) in the DG were examined after every session of behavioral test; (b) control+vehicle, SD+vehicle and SD+isoproterenol (SD+ISO) groups: everyday before performing behavioral tests, isoproterenol or vehicle was microinjected into the DG region, and fEPSP amplitudes were examined after the behavioral test. The DG tissue was separated and collected under the microscope at the end of the behavioral experiments.

\section{Sleep deprivation}

As Fig. 1 shown, animals were sleep-deprived for $18 \mathrm{~h}$ per day for 21 consecutive days by placing each animal in a device. The deprivation rod in the device can slide periodically around the bottom of the device (XR-XS107, Shanghai xinruan, China). The mobile programmed on a repeated cycle of $30 \mathrm{~s}$ on $(3 \mathrm{~m} / \mathrm{min})$ and $2 \mathrm{~min}$ off during SD. Food and water were available throughout the SD
A

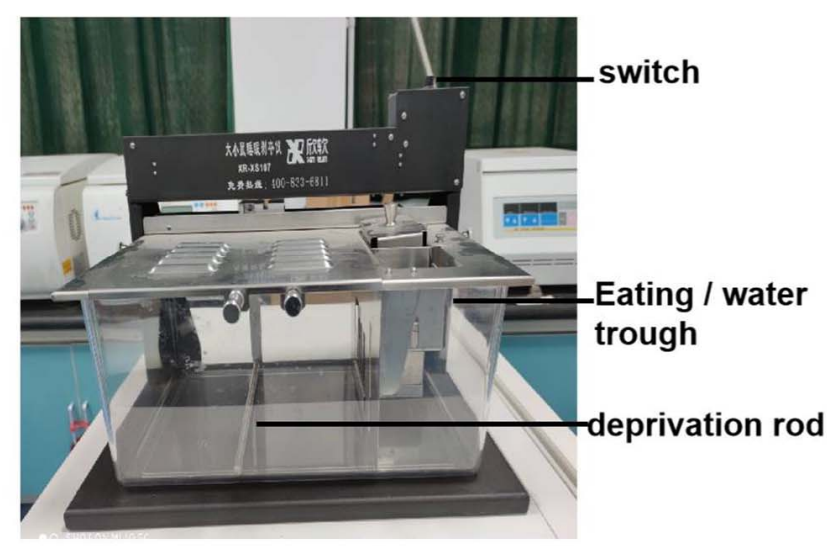

B

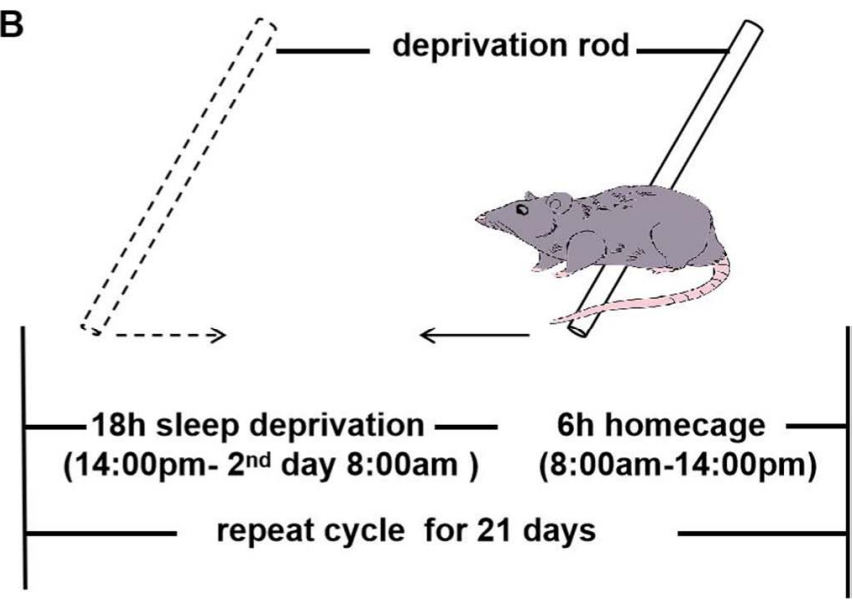

Fig. 1. Sleep-deprived device (A) and sleep deprivation process (B). 
period.

\section{Reagents and antibodies}

Norepinephrine, isoproterenol ( $2 \mu \mathrm{g} / \mu \mathrm{l}$ dissolved in saline) and EDTA-2Na were purchased from Sigma. 30 min prior to everyday behavioral training, the isoproterenol was injected into the hippocampal DG area at a speed of $0.5 \mu \mathrm{l} / \mathrm{min}$, volume $1 \mu \mathrm{l}$ and the duration of administration was $2 \mathrm{~min}$. N-methyl-D-aspartate (NMDA) Receptor 1 (GluN1) Rabbit mAb (\#5704S), a-amino-3hydroxy-5-methylisoxazole-4-propionic acid (AMPA) receptor (GluA2/3/4) Antibody (\#2460S), Anti-rabbit IgG, HRP-linked Antibody (\#7074) and $\beta$-Actin (8H10D10) Mouse mAb (\#3700) were purchased from Cell signaling.

\section{Experimental procedures}

Rats were anesthetized with $10 \%$ chloral hydrate $(300 \mathrm{mg} / \mathrm{kg}$ intraperitoneally) and placed on a stereotaxic frame (DW-2000, Chengdu taimeng, China). A guide cannula affixed with on a 20 G stainless steel tubing (Terumo, Tokyo, Japan) was implanted $1.5 \mathrm{~mm}$ above the DG region (bregma as a reference point, AP $3.2 \sim 3.4 \mathrm{~mm}, \mathrm{~L} / \mathrm{R} 1.8 \sim 2.0 \mathrm{~mm}, \mathrm{H} 2.5 \mathrm{~mm}$ ) and a bipolar stimulating electrode (A-M Systems, Sequim, USA) was lowered into the ipsilateral central perforant pathway (PP, bregma as a reference point, AP $6.8 \sim 7.0 \mathrm{~mm}, \mathrm{~L} / \mathrm{R} 4.9 \sim 5.1 \mathrm{~mm}, \mathrm{H} 4.5 \sim 5.0 \mathrm{~mm}$ ) as previously described [19]. The location of cannula and electrodes were shown in Fig. 2.

All the animals were allowed to recover from surgery for 3 days. On the day before the experiment, a microdialysis probe or microinjection tube was inserted through the guide cannula into the DG region and stabilized with wax. To reach the DG region, the tip of the microdialysis probe, covered with $1.0 \mathrm{~mm}$-long hollow fibers $\left(200 \mu \mathrm{m}\right.$ outer diameter, cutoff $5.0 \times 10^{4}$ molecular weight; Eicom, Kyoto, Japan) was set to extend $1.0 \mathrm{~mm}$ beyond the guide shaft. A monopolar recording electrode (A-M Systems) inserted into the $20 \mathrm{G}$ stainless steel tubing reached the DG region, where extracellular field potentials evoked by stimulation of the PP were recorded. The recording electrode was lowered until the maximal evoked response was visually confirmed, and then fixed by dental cement.

\section{Measure of concentrations of NE and fEPSP}

On the day of experimentation, NE levels and fEPSP amplitudes were measured under freely moving conditions as described in previously published protocols [19]. Briefly, the microdialysis probe was perfused with modified Ringer solution at $1.0 \mu \mathrm{l} / \mathrm{min}$ using a micro-infusion pump (ESP-64; Eicom, Japan) $40 \mathrm{~min}$ after MWM test every day, and three consecutive dialysate samples were collected. Then the dialysate from the DG region was injected into the high-performance liquid chromatography and electrochemical detection system (ECD-300; Eicom) to measure the concentrations of NE. The composition of the mobile phase was $0.1 \mathrm{M}$ PBS (pH 6.0), 5\% methanol, $50 \mathrm{mg} / \mathrm{L}$ EDTA-2Na, and 500 $\mathrm{mg} / \mathrm{L}$ sodium 1-octanesulfonate.

Measurement of fEPSP amplitudes was performed after MWM test 10 min, the PP was stimulated 15 times by single-phase square wave pulses $(0.1 \mathrm{~ms} /$ phase, intensity chosen to elicit $50 \%$ of the maximal fEPSP, intensity from $0.01 \mathrm{~mA}$ to $0.3 \mathrm{~mA}$, interval $30 \mathrm{~s}$ ) generated with the flexible stimulus isolator (ISO-Flex, AMPI, Jerusalem, Israel). Evoked responses were filtered $(0.5 \sim 2.0 \mathrm{kHz})$ and amplified $(\times 1,000)$ by an AC amplifier (NeuroLog; Digitimer, Welwyn Garden City, Hertfordshire, UK), digitized (Micro3; CED, Cambridge, UK), and analyzed on a computer with Spike2 software (CED). Overall, 15 fEPSP traces were averaged to obtain the mean amplitude.

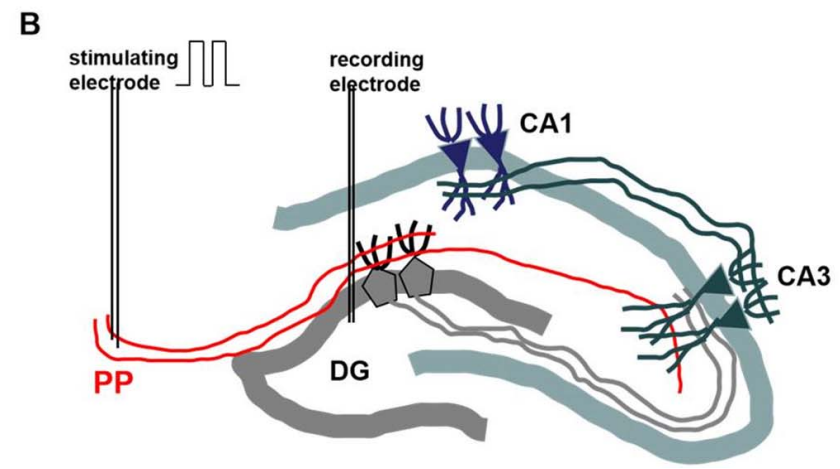

Fig. 2. The location of cannula, recording electrode (A) and stimulating electrode (B). 


\section{Morris water maze (MWM) test}

Spatial L\&M abilities of rats were assessed by MWM test on 9:00 12:00 am every day. The MWM consists of four parts: circular pool, platform, automatic recording system and water supply system. Among them, the water maze pool diameter is $170 \mathrm{~cm}$, high $60 \mathrm{~cm}$. The cylindrical platform diameter is $10 \mathrm{~cm}$ and high $40 \mathrm{~cm}$. Surface of the water pool during testing is about $45 \mathrm{~cm}$. Room temperature and water temperature at $22 \pm 2^{\circ} \mathrm{C}$, the platform is placed in the center of third quadrant. The MWM test include place navigation trials ( $1^{\text {st }}$ to $4^{\text {th }}$ days) and spatial probe trial $\left(5^{\text {th }}\right.$ day). In the first 4 days, rats experienced four trials per day. In each trial, rats were allowed to swim freely for $120 \mathrm{~s}$ to find the hidden platform. The time they spent finding and standing on the platform with all limbs was considered the escape latency. If the rats cannot find the platform, they were guided to the platform and the escape latency was recorded as $120 \mathrm{~s}$. In the $5^{\text {th }}$ day of the probe test, circular platforms were removed from the pool, and animals were placed in the water at a given location to swim freely for $120 \mathrm{~s}$. The proportion of total time spent in each quadrant and the number of platform crossings were recorded.

\section{Western blot analysis}

The expression of glutamatergic receptors in the DG region was measured by Western blot. After all the experiments, the animal was anesthetized to death and the hippocampal DG area was separated under microscope. RIPA lysis buffer was used to extract the protein samples (sample $20 \mathrm{mg}$, lysate 150 250 $\mu$ l, PMSF 1.5 2.5 $\mu \mathrm{l})$. Samples $(10 \mu \mathrm{g})$ were then separated by $12 \%$ SDS-PAGE, transferred to PVDF membranes and incubated with 5\% skim milk at room temperature. The membranes were incubated overnight at $4{ }^{\circ} \mathrm{C}$ with GluN1 and GluA2/3/4 antibodies. After washing, they were incubated with the corresponding secondary antibodies for $2 \mathrm{~h}$ at room temperature. Expression levels were measured by enhanced chemiluminescence (ECL, Pierce Biotechnology, USA). The experiment was performed in triplicates on at least three occasions.

\section{Statistical analysis}

All data are expressed as mean \pm SEM and were analysed using SPSS 19.0 software. The NE levels, fEPSP amplitude, swimming speed and distance and number of platform crossings between two groups were calculated through independent-samples t-test. The escape latency between two groups was using two-way ANOVA. Other data were analysed using a one-way ANOVA followed by the Dunnett test or repeated measures. A value of $\mathrm{p}<0.05$ was considered statistically significant.

\section{RESULTS}

\section{SD impairs spatial L\&M in rats}

Spatial L\&M performance was evaluated by the MWM test. As Fig. 3A shown, during the place navigation trial, SD group rats performed in the MWM as well as their control group, exhibiting rapidly decreasing escape latencies $\left(\mathrm{F}_{(3,47)}=16.476, \mathrm{p}=0.000\right)$. There was significant different between two groups $\left(\mathrm{F}_{(1,47)}=11.690\right.$, $\mathrm{p}=0.001)$. However, there was no interaction between group and training day $\left(\mathrm{F}_{(3,47)}=1.962, \mathrm{p}=0.135\right)$.

On the spatial probe trial of day 5 , rats in the control group showed a significant bias for the target quadrant where the platform had been originally located (Fig. $3 \mathrm{C}$ and $\mathrm{F}, \mathrm{F}_{(4,25)}=3.737$, $\mathrm{p}=0.016)$. However, rats in SD group failed to show preference for the target quadrant (Fig. 3C and $\mathrm{F}_{(4,25)}=0.987, \mathrm{p}=0.433$ ). The number of platform crossings was significantly decreased when compared to the control (Fig. 3B, $\mathrm{t}=2.345, \mathrm{p}=0.041$ ). However, there were no significant different in time spent in the target quadrant (Fig. 3C, $\mathrm{t}=2.122, \mathrm{p}=0.060$ ), total swimming distance (Fig. 3D, $\mathrm{t}=2.524, \mathrm{p}=0.612$ ) and swimming speed (Fig. 3E, $\mathrm{t}=-0.252$, $\mathrm{p}=0.806$ ) between control and SD groups in day 5 .

\section{SD decreases NE concentration and synaptic efficiency in DG during spatial L\&M}

In the present study, the 'basal levels' indicate the values obtained before starting behavioral test in each parameter. The basal levels of NE in the SD group and control group were $0.114 \pm 0.035 \mathrm{pg} / \mu \mathrm{l}$ and $0.122 \pm 0.023 \mathrm{pg} / \mu \mathrm{l}$ respectively, and the difference between the two groups was not significant $(\mathrm{t}=0.189, \mathrm{p}=0.854)$.

The extracellular concentrations of NE in the DG region in control group were significantly increased during MWM test (Fig. 4A and $\left.\mathrm{B}, \mathrm{F}_{(5,30)}=16.619, \mathrm{p}=0.000\right)$. $\mathrm{SD}$ group rats also exhibit elevation of NE during the MWM test (Fig. $4 \mathrm{~A}$ and $\mathrm{B}, \mathrm{F}_{(5,30)}=8.687, \mathrm{p}=0.000$ ), but the magnitude of elevation was lower than control group on day 2, day 3 and day 5 (Fig. $4 \mathrm{~B}, \mathrm{t}_{1 \mathrm{st}}=1.907, \mathrm{p}_{1 \mathrm{st}}=0.086$; $\mathrm{t}_{2 \mathrm{nd}}=2.629$, $\mathrm{p}_{2 \mathrm{nd}}=0.025 ; \mathrm{t}_{3 \mathrm{rd}}=3.425, \mathrm{p}_{3 \mathrm{rd}}=0.006 ; \mathrm{t}_{4 \mathrm{th}}=1.332, \mathrm{p}_{4 \mathrm{th}}=0.213 ; \mathrm{t}_{5 \mathrm{th}}=3.863$, $\left.\mathrm{p}_{5 \mathrm{th}}=0.003\right)$.

To examine the synaptic efficacy in the DG region, the fEPSP amplitude was measured synchronously. There was no significant different in the basal fEPSP amplitude between two groups (Fig. $4 \mathrm{C}$ and $\mathrm{D}, \mathrm{t}=0.825, \mathrm{p}=0.429$ ). In the MWM test, the fEPSP amplitude in DG in the control group was enhanced following the training session (Fig. $4 \mathrm{C}$ and $\mathrm{E}, \mathrm{F}_{(5,30)}=5.581, \mathrm{p}=0.001$ ). In contrast, the fEPSP amplitude in the SD group was not changed during MWM test (Fig. 4E, $\left.\mathrm{F}_{(5,30)}=2.016, \mathrm{p}=0.105\right)$. In addition, the fEPSP amplitude in DG in SD group was significantly decreased on the $3^{\text {rd }}$ and $4^{\text {th }}$ day compared with control group (Fig. $4 \mathrm{E}, \mathrm{t}_{1 \mathrm{st}}=1.167, \mathrm{p}_{1 \mathrm{st}}=0.270$; 

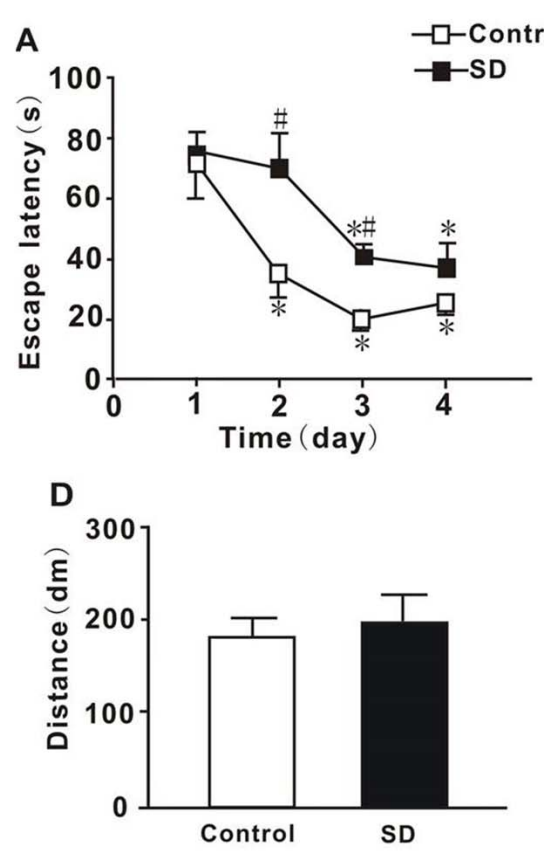

$\mathbf{B}$
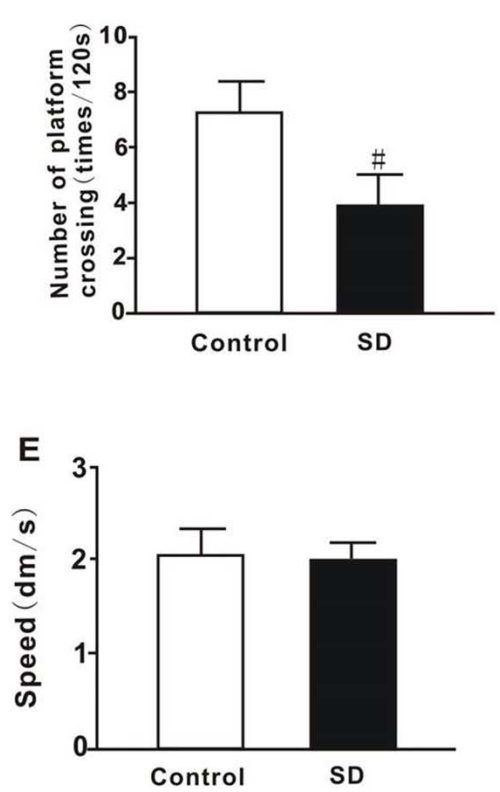

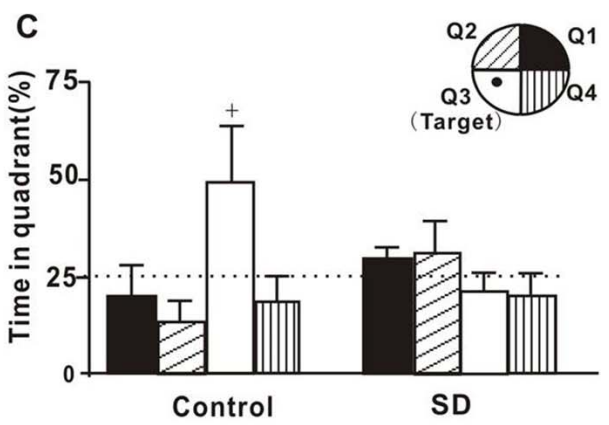

$\mathbf{F}$

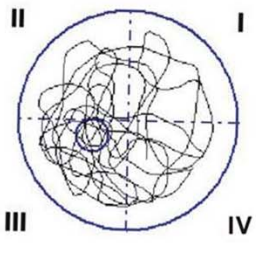

Control

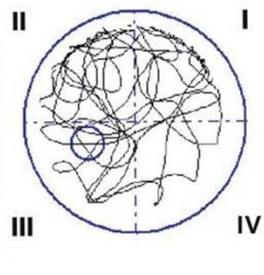

SD

Fig. 3. Effects of SD on spatial L\&M. (A) The escape latency in the place navigation trial of MWM test, (B) the number of platform crossing, (C) the proportion of total time spent in each quadrant, (D) the total swimming distance, (E) swimming speed and (F) representative swimming traces in the spatial probe trial of MWM test. Data are presented as mean \pm SEM ( $\mathrm{n}=6$ in each group). ${ }^{*} \mathrm{p}<0.05$ compared to day $1 ;{ }^{*} \mathrm{p}<0.05$ compared to the control group; ${ }^{+} \mathrm{p}<0.05$ compared to $25 \%$ chance level in each quadrant. $\mathrm{dm}$, decimeter; $\mathrm{dm} / \mathrm{s}$, decimeter/second.
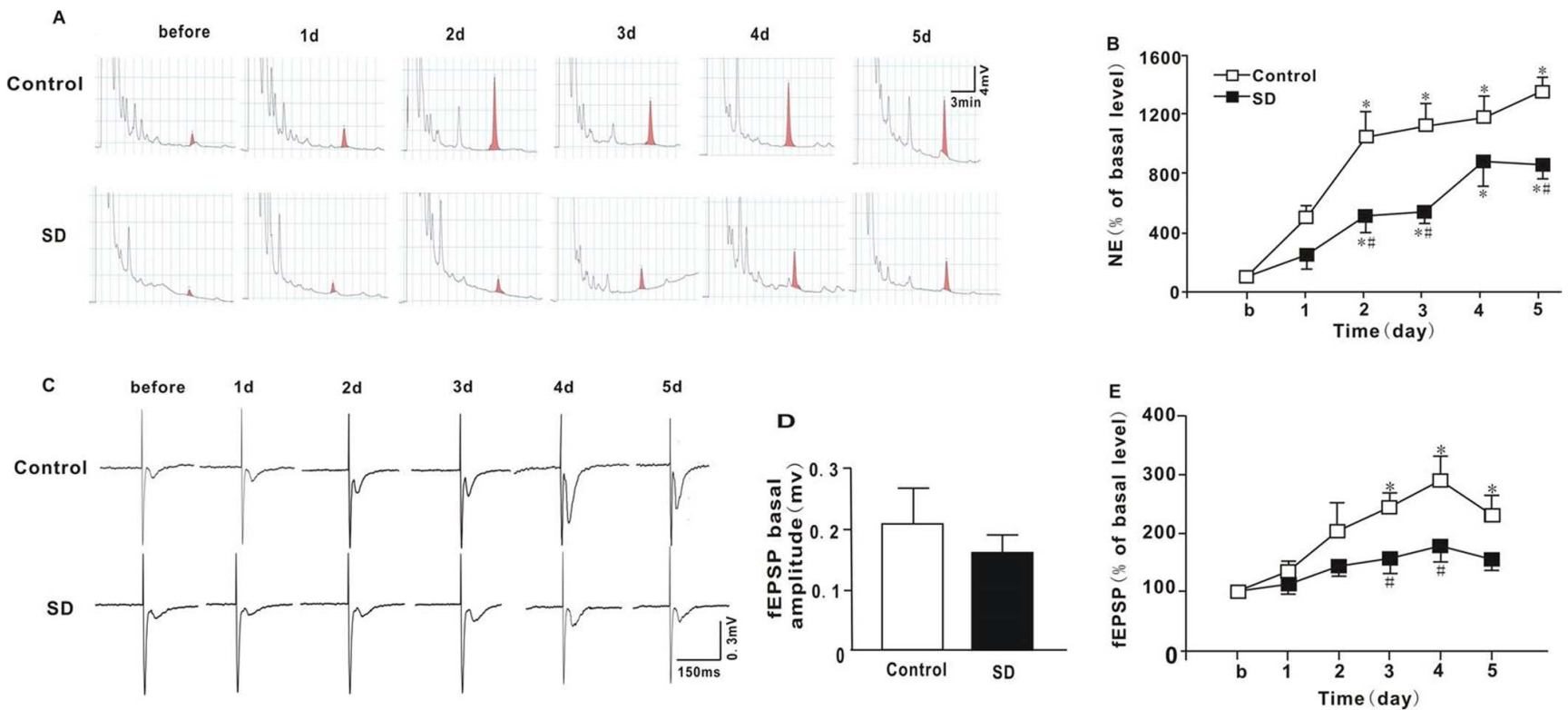

Fig. 4. Effects of SD on NE concentration and fEPSP amplitude in hippocampal DG region during MWM test. (A) Everyday typical HPLC-ECD chromatogram of the NE during MWM test, (B) Summary of changes of NE level, (C) Everyday typical fEPSP waveform in the DG during MWM test, (D) Basal fEPSP amplitude and (E) Amplitude of fEPSP in DG in SD rats during MWM test. 'b' indicates before starting the behavioral test. NE levels and fEPSP amplitudes are expressed as percentage of basal levels. Data are presented as mean \pm SEM ( $n=6$ in each group). ${ }^{*} \mathrm{p}<0.05$ vs. basal level. ${ }^{*} \mathrm{p}<0.05 \mathrm{vs}$. control group. fEPSP, field excitatory postsynaptic potential; NE, norepinephrine. 

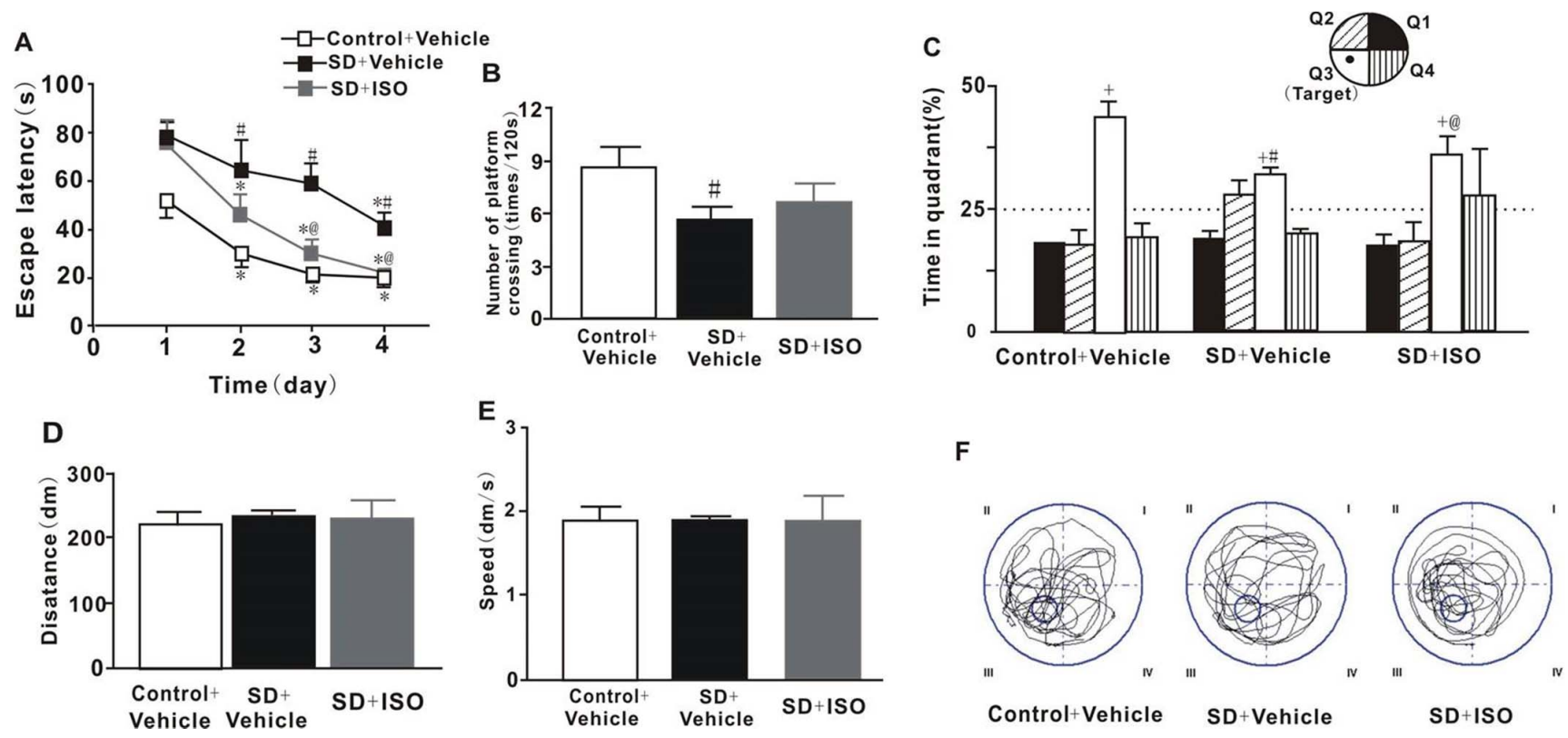

Fig. 5. Effects of isoproterenol on spatial L\&M of SD rats. (A) The escape latency in the place navigation trial of MWM test, (B) the number of platform crossing, (C) the proportion of total time spent in each quadrant, (D) the total swimming distance, (E) swimming speed and (F) representative swimming traces in the spatial probe trial of MWM test. Data are presented as mean $\pm \mathrm{SEM}$ ( $\mathrm{n}=6$ in each group). ${ }^{*} \mathrm{p}<0.05$ compared to day $1 ;{ }^{*} \mathrm{p}<0.05 \mathrm{vs}$. Control+vehicle group; ${ }^{\oplus} \mathrm{p}<0.05$ vs. SD+vehicle group; ${ }^{+} \mathrm{p}<0.05 \mathrm{vs} .25 \%$ chance level in each quadrant. $\mathrm{dm}$, decimeter; $\mathrm{dm} / \mathrm{s}$, decimeter/second.

$\mathrm{t}_{2 \mathrm{nd}}=1.137, \mathrm{p}_{2 \mathrm{nd}}=0.282 ; \mathrm{t}_{3 \mathrm{rd}}=2.424, \mathrm{p}_{3 \mathrm{rd}}=0.036 ; \mathrm{t}_{4 \mathrm{th}}=2.365, \mathrm{p}_{4 \mathrm{th}}=0.040$; $\left.\mathrm{t}_{5 \mathrm{th}}=2.040, \mathrm{p}_{5 \mathrm{th}}=0.069\right)$. These results suggest that changes in $\mathrm{NE}$ level in the DG were accompanied by changes in fEPSP, consistent with the behavioral results.

\section{Isoproterenol improved spatial L\&M impairments in SD rats}

To further investigate whether the increase of NE in the DG is involved in spatial L\&M impairment of SD rats by $\beta$-AR, isoproterenol (an agonist of $\beta$-AR) was microinjected into the DG, and the spatial L\&M were investigated. As shown in Fig. 5A, the escape latency gradually lower over the course of the 4 training days of the place navigation trial in control+vehicle group $\left(\mathrm{F}_{(3,20)}=8.901\right.$, $\mathrm{p}=0.001)$, in $\mathrm{SD}+$ Vehicle group $\left(\mathrm{F}_{(3,20)}=3.212, \mathrm{p}=0.045\right)$ and in $\mathrm{SD}+\mathrm{ISO}$ group $\left(\mathrm{F}_{(3,20)}=8.993, \mathrm{p}=0.001\right)$, suggesting that the learning process was intact in all rats. However, in SD+ISO group, the escape latency on the $3^{\text {rd }}$ and $4^{\text {th }}$ day was significantly reduced compared with SD+vehicle group (Fig. $5 \mathrm{~A}, \mathrm{~F}_{(2,15)}=11.352$, $\mathrm{p}=0.001$ in day $3, \mathrm{~F}_{(2,15)}=5.793, \mathrm{p}=0.014$ in day 4$)$, showing improvement in spatial learning.

In the spatial probe trial, rats in all group showed a significant bias for the target quadrant where the platform had been originally located (Fig. 5C and F, $\mathrm{F}_{(4,25)}=18.487, \mathrm{p}=0.000$ in control+Vehicle, $\mathrm{F}_{(4,25)}=11.963, \mathrm{p}=0.000$ in $\mathrm{SD}+$ Vehicle, $\mathrm{F}_{(4,25)}=3.276, \mathrm{p}=0.027$ in
SD+ISO). Compared with SD+vehicle group, microinjection of isoproterenol into the DG significantly increased the percentage of time spent in target quadrant (Fig. 5 C, $\mathrm{F}_{(2,15)}=3.954$, $\mathrm{p}=0.042$ ), although the differences were not statistically significant in the number of platform crossings (Fig. $5 \mathrm{~B}, \mathrm{~F}_{(2,15)}=2.3$, $\mathrm{p}=0.135$ ), total swimming distance (Fig. 5D, $\left.\mathrm{F}_{(2,15)}=0.052, \mathrm{p}=0.949\right)$ and swimming speed (Fig. 5E, $\mathrm{F}_{(2,15)}=0.029$, $\mathrm{p}=0.971$ ).

\section{Isoproterenol increases synaptic efficiency in DG in SD rats}

The effects of a microinjection of isoproterenol on fEPSP amplitudes in the hippocampal DG during MWM test are shown in Fig. 6. In all groups, the basal fEPSP amplitude have no significant different (Fig. 6A and B, $\mathrm{F}_{(2,15)}=1.701, \mathrm{p}=0.216$ ). The fEPSP amplitude in $\mathrm{DG}$ were markedly enhanced as the experiment progressed (Fig. 6A and $\mathrm{C}, \mathrm{F}_{(5,30)}=3.928, \mathrm{p}=0.007$ in control+vehicle group; $\mathrm{F}_{(5,30)}=5.665, \mathrm{p}=0.001$ in SD+vehicle group; $\mathrm{F}_{(5,30)}=11.941, \mathrm{p}=0.000$ in SD+ISO group). Nevertheless, compared with SD+vehicle group, the fEPSP amplitude in DG in SD+ISO group was significantly lower from day 2 to day $5\left(\mathrm{~F}_{(2,15)}=4.174, \mathrm{p}=0.036\right.$ in day $2 ; \mathrm{F}_{(2,15)}=7.538, \mathrm{p}=0.005$ in day $3 ; \mathrm{F}_{(2,15)}=8.779, \mathrm{p}=0.003$ in day 4; $\mathrm{F}_{(2,15)}=3.995, \mathrm{p}=0.041$ in day 5$)$. 

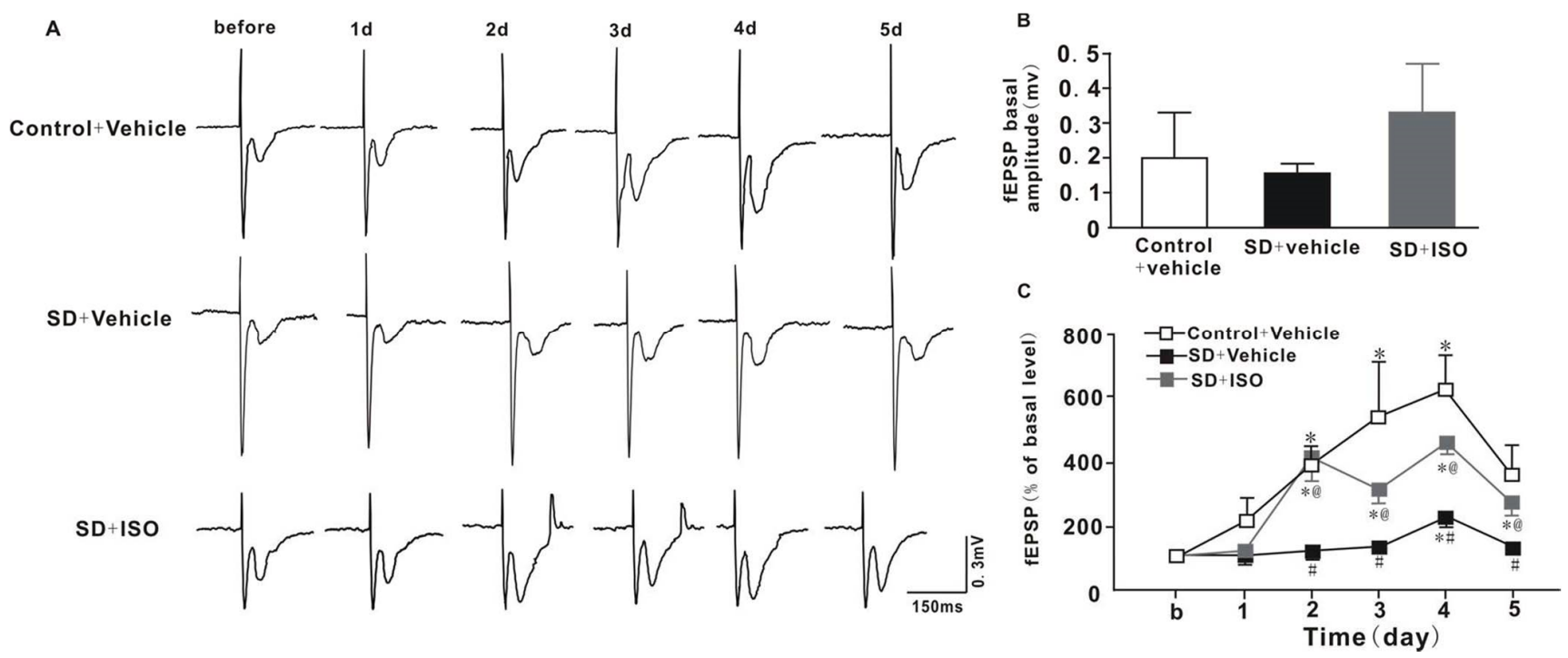

Fig. 6. Effects of isoproterenol on fEPSP amplitude in hippocampal DG region of SD rats during MWM test. (A) Everyday typical fEPSP waveform in the DG during MWM test, (B) Basal fEPSP amplitude and (C) Amplitude of fEPSP in DG in SD rats during MWM test. 'b' indicates before starting the behavioral test. fEPSP amplitudes are expressed as percentage of basal levels. Data are presented as mean \pm SEM ( $\mathrm{n}=6$ in each group). ${ }^{*} \mathrm{p}<0.05 \mathrm{vs}$. basal level. ${ }^{*} \mathrm{p}<0.05$ vs. Control+vehicle group; ${ }^{\oplus} \mathrm{p}<0.05$ vs. $\mathrm{SD}+$ Vehicle group. $\mathrm{fEPSP}$, field excitatory postsynaptic potential.

\section{Isoproterenol increases glutamatergic receptors expression level in DG in SD rats}

In this study, Western blotting analysis was used for evaluating the expression level of glutamatergic receptors, including NMDA and AMPA receptor in the DG region. The expression level of GluN1 and GluA2/3/4 in SD rats was obviously reduced compared with control rats. Interestingly, treatment with isoproterenol resulted in a significant increase of GluN1 and GluA2/3/4 protein levels when compared to the $\mathrm{SD}+$ vehicle groups (Fig. 7, $\mathrm{F}_{(2,15)}=6.129, \mathrm{p}=0.011$ in GluN1; $\mathrm{F}_{(2,15)}=6.883, \mathrm{p}=0.008$ in GluA2/3/4), suggesting that activation of $\beta$-AR in the DG region promotes glutamatergic receptors levels.

\section{DISCUSSION}

It has been known that lack of sleep has a detrimental impact on cognitive functions. Various studies have emphasized the impairment of SD on L\&M, particularly the types mediated by the hippocampus, such as spatial L\&M $[3,21]$. In the present study, we reported that SD rats displayed a similar deficit in spatial L\&M, based on the fact that the escape latencies (representing the learning ability) were prolonged and the percentage of time spent in target quadrant and the number of platform crossings (reflects the maintenance of memory function) was reduced in the MWM test.

The hippocampus consists of distinct subregions that each contribute differently to the memory consolidation process. Several studies have shown that DG plays a critical role in L\&M. Previous studies which tested these models using selective lesions of hippocampal subregions suggest that the DG region is crucial for encoding and processing spatial information [12-14]. LTP is a cellular model for information processing and memory storage that has been used to study the impact of SD on L\&M $[3,5]$. As mentioned above, a major input to the DG arises from the entorhinal cortex via the PP, and the expression of LTP in this pathway varies with the learning state [22]. Our results have shown that in vivo induction of long-term enhancement on synaptic efficiency correlated with the rat ability to perform a shuttle-box avoidance task [19]. Therefore, the LTP recorded in DG can be considered as learningdependent. In the present study, the fEPSP amplitudes in the DG in the control group increased gradually with the increase of training days in rats in MWM test, and the changes were accompanied by the acquisition of spatial L\&M suggesting that the LTP observed in the present study was LD-LTP. In addition, we found that the LD-LTP in hippocampal DG was significantly suppressed in the SD group, which may contribute to the disruption of spatial L\&M functions.

The noradrenergic system, driven by LC activation, plays a key role in the regulating and directing of changes in hippocampal $\mathrm{L} \& \mathrm{M}$ processes and synaptic plasticity. In addition, the stimulation of PP not only affect the induction of LTP but also extracellular concentration of NE in DG region [23, 24]. Behavioral studies show that LC stimulation or intrahippocampal NE injection 
A

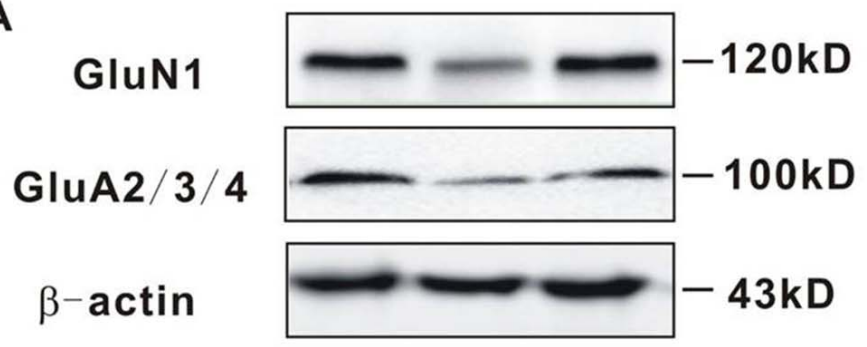

B

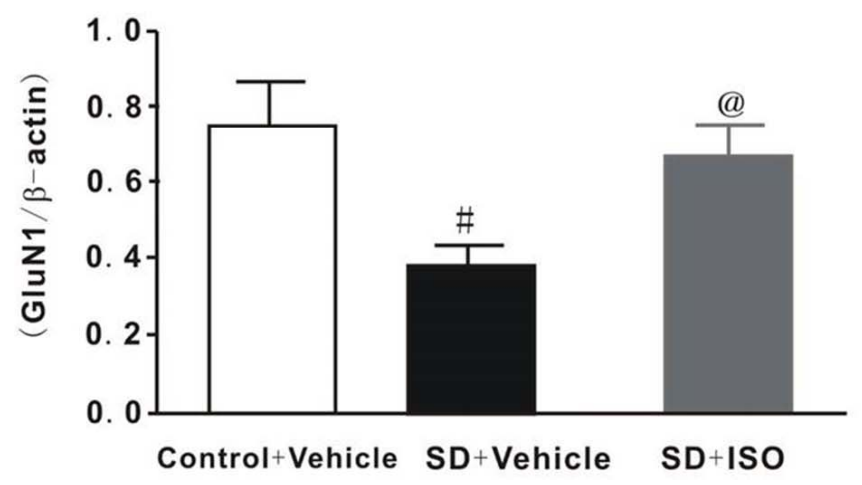

C

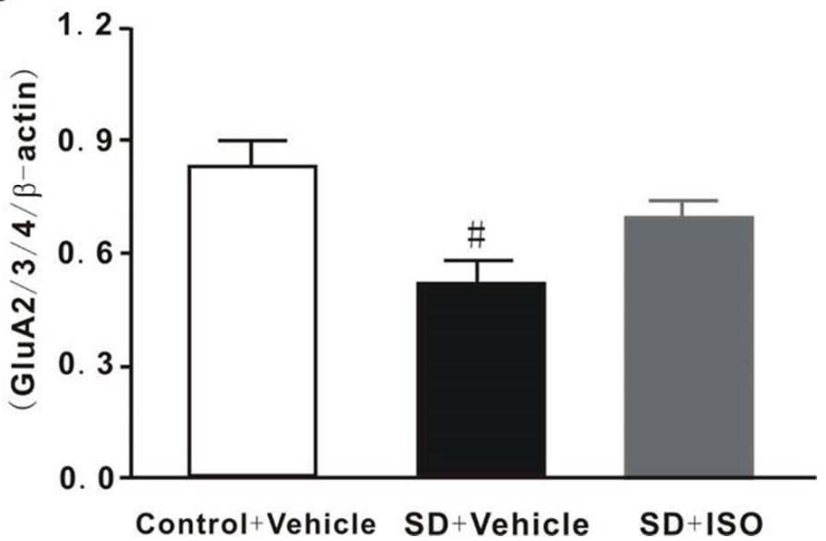

Fig. 7. Effects of isoproterenol on GluN1 (A, B) and GluA2/3/4 (A, C) level in the hippocampal DG region of SD rats. Data are presented as mean \pm SEM ( $\mathrm{n}=6$ in each group). ${ }^{*} \mathrm{p}<0.05$ vs. Control+vehicle group; ${ }^{\circledR} \mathrm{p}<0.05$ vs. SD+Vehicle group.

promotes retrieval of spatial and associative memories $[25,26]$. Moreover, NE release after LC activation is essential for induction and maintenance of LTP in the DG of freely behaving rats, and it may be regarded as a basis for the formation and consolidation of memory, all of which requires activation of $\beta$-AR $[18,20,27]$. Consistent with these studies, our results exhibited that the extracellular concentration of NE in two groups in the DG significantly increased during place navigation trial and subsequent spatial probe trial, and the maximum NE levels in control and SD group were on $5^{\text {th }}$ and $4^{\text {th }}$ day, respectively. Moreover, NE level in the DG was decreased in SD rats compared with control rat, indicating that NE in the DG region might be involved in spatial L\&M impairments in SD. Interestingly, our results showed that the changes of NE levels were accompanied by changes in the fEPSP amplitude in the same region, because of the maximum amplitude of fEPSP between control and SD groups were all on day 4. It is well known that NE has a powerful effect on L\&M and it is essential for induction and maintenance of LTP in the DG. Therefore, the change of maximum NE level in this study may be correlate with L\&M and the enhancement of synaptic plasticity. These results suggesting that NE in the hippocampal DG region is involved in LD-LTP during spatial L\&M in SD.

Many studies have shown that NE can not only enhance memory formation, but also has a powerful effect on the induction of LTP throughout the hippocampus, and this process is mainly mediated by $\beta$-AR [28]. The highest expression level of $\beta$-ARs is found in the DG [17], mostly within granule cells at postsynaptic sites. Studies found that $\beta$-AR-blockade in CA1 leads to deficits in spatial reference memory in the water maze task [29]. Furthermore, learning-facilitated LTP is prevented by $\beta$-AR antagonism, as well as spatial learning of novel empty space in the DG of freely behaving rats [20]. Our previous results exhibited that microinjection of the $\beta$-AR antagonist (propranolol) into DG inhibited the active avoidance learning, whereas microinjection of isoproterenol facilitated the active avoidance learning [19]. We therefore speculate that the $\beta$-AR in the DG is involved in spatial L\&M and might be related to LD-LTP impairment by SD. In present results, we found that both the ability of spatial L\&M and LD-LTP were significantly depressed in SD rats. However, the activation of $\beta$-AR by isoproterenol in the DG significantly attenuated the impairment of LD-LTP and subsequently spatial L\&M deficits in SD rats. It has been shown that $\beta$-AR antagonism blocks the depolarization [30] 
and $\beta$-AR agonism increases the activity of voltage-dependent calcium channels in granule cell of the DG [31]. Thus, elevation of NE levels increases neuronal excitability in the DG via activation of $\beta$-AR. Therefore, we speculate that $\beta$-ARs in the DG are involved in spatial cognitive and related to LD-LTP impairment of SD by regulating the excitability of the principal cells.

It has been shown that the induction of LD-LTP in the hippocampal DG regions is typically dependent on the activation of NMDA-type glutamate receptors $[32,33]$. In the hippocampus, the activation of $\beta$-AR enhances NMDA receptor-mediated $\mathrm{Ca}^{2+}$ transients by cAMP-dependent protein kinase (PKA) [20]. Given the central role of NMDA receptor activation in the induction of LTP, this suggests that modulation of NMDA receptor function has a pivotal role in the ability of $\beta$-AR activation to enhance LTP induction. NMDA receptors are unique among neurotransmitter receptors because their activation depends on glutamate binding. Previous results have shown that agonism of $\beta$-AR in the hippocampal DG region increased presynaptic local release of glutamate and simultaneously facilitated the induction and maintenance of LD-LTP [19]. Therefore, we concluded that $\beta$-AR in the DG facilitate the spatial learning process and LD-LTP by increasing the activation of many synaptic plasticity-related proteins including NMDA receptor. Our study showed that the levels of GluN1 in the DG significantly decreased in SD rats, however, the activation of $\beta$-AR increases GluN1 expression. The results suggest that $\beta$-AR in the DG region are involved in spatial learning, which may act via NMDA receptor to induce the learning associated LTP. In recent years, research has suggested $\beta$-AR activation could also facilitate NMDA receptors activation by enhancing membrane depolarization at active synapses [30]. Thus, these results providing a mechanism whereby $\beta$-AR activation can boost spine depolarization at active synapses and facilitate LTP induction by enhancing NMDA receptor activation.

In addition to NMDA receptor, AMPA receptor, another type of glutamate receptors, also play active roles in controlling both plasticity induction and expression in the hippocampus $[28,34]$. Studies found that $\beta$-AR activation induce GluA1 phosphorylation through PKA in hippocampal neurons [35]. However, in mice with knock-out specific GluA1 phosphorylation sites, the enhancement of contextual fear learning by systemic epinephrine injections and enhancement of LTP by $\beta$-AR were disrupted [11], suggesting that AMPA receptor has a key role in the enhancement of L\&M as well as in $\beta$-AR modulation of LTP. Therefore, we investigated whether the involvement of $\beta$-AR in SD rats spatial learning is related to the expression of AMPA in the DG. Our results showed that $\beta$-AR activation enhances the level of GluA2/3/4 in DG. Recent studies have implicated that AMPA receptor can be as another $\mathrm{Ca}^{2+}$ source in additional to $\mathrm{Ca}^{2+}$ mediated by NMDA receptor [36]. Furthermore, trafficking of AMPA receptors from this extrasynaptic pool into synapses is suggested to have a crucial role in LTP [37]. Thus, these results suggesting that $\beta$-AR activation enhances LD-LTP, at least in part, by increasing release mediated by AMPA or the pool of membrane AMPA receptors that are available for synaptic insertion following LTP induction.

In conclusion, the findings of the present study suggest that activation of $\beta$-AR in the hippocampal DG improves spatial L\&M impairments in $\mathrm{SD}$ rats by enhancing synaptic plasticity and glutamatergic receptors expression.

\section{ACKNOWLEDGEMENTS}

We are grateful for the financial supports from Scientific Research Foundations of Education Department of Hebei Province(QN2020158) for Dr. Jing lv and Nantong University Grants for Dr. Huan-Jun Lu.

\section{REFERENCES}

1. Meerlo P, Havekes R, Steiger A (2015) Chronically restricted or disrupted sleep as a causal factor in the development of depression. Curr Top Behav Neurosci 25:459-481.

2. Han KS, Kim L, Shim I (2012) Stress and sleep disorder. Exp Neurobiol 21:141-150.

3. Peng Y,Wang W, Tan T, He W, Dong Z, Wang YT, Han H (2016) Maternal sleep deprivation at different stages of pregnancy impairs the emotional and cognitive functions, and suppresses hippocampal long-term potentiation in the offspring rats. Mol Brain 9:17.

4. Walker MP (2008) Cognitive consequences of sleep and sleep loss. Sleep Med 9 Suppl 1:S29-S34.

5. Wadhwa M, Prabhakar A, Ray K, Roy K, Kumari P, Jha PK, Kishore K, Kumar S, Panjwani U (2017) Inhibiting the microglia activation improves the spatial memory and adult neurogenesis in rat hippocampus during $48 \mathrm{~h}$ of sleep deprivation. J Neuroinflammation 14:222.

6. Havekes R, Park AJ, Tudor JC, Luczak VG, Hansen RT, Ferri SL, Bruinenberg VM, Poplawski SG, Day JP, Aton SJ, Radwańska K, Meerlo P, Houslay MD, Baillie GS, Abel T (2016) Sleep deprivation causes memory deficits by negatively impacting neuronal connectivity in hippocampal area CA1. Elife 5:e13424.

7. Bliss TV, Collingridge GL (1993) A synaptic model of memory: long-term potentiation in the hippocampus. Nature 361:31-39. 
8. Choi JE, Kim S, Lee J, Kim K, Kaang BK (2018) Circadian regulation by REV-ERBa mediates hippocampal E-LTP in a time-dependent manner. Exp Neurobiol 27:344-349.

9. Marks CA, Wayner MJ (2005) Effects of sleep disruption on rat dentate granule cell LTP in vivo. Brain Res Bull 66:114119.

10. Süer C, Dolu N, Artis AS, Sahin L, Yilmaz A, Cetin A (2011) The effects of long-term sleep deprivation on the long-term potentiation in the dentate gyrus and brain oxidation status in rats. Neurosci Res 70:71-77.

11. Hu H, Real E, Takamiya K, Kang MG, Ledoux J, Huganir RL, Malinow R (2007) Emotion enhances learning via norepinephrine regulation of AMPA-receptor trafficking. Cell 131:160-173.

12. Aimone JB, Deng W, Gage FH (2011) Resolving new memories: a critical look at the dentate gyrus, adult neurogenesis, and pattern separation. Neuron 70:589-596.

13. Gilbert PE, Kesner RP, Lee I (2001) Dissociating hippocampal subregions: double dissociation between dentate gyrus and CA1. Hippocampus 11:626-636.

14. Kesner RP (2018) An analysis of dentate gyrus function (an update). Behav Brain Res 354:84-91.

15. Dommett EJ, Henderson EL, Westwell MS, Greenfield SA (2008) Methylphenidate amplifies long-term plasticity in the hippocampus via noradrenergic mechanisms. Learn Mem 15:580-586.

16. Sara SJ (2009) The locus coeruleus and noradrenergic modulation of cognition. Nat Rev Neurosci 10:211-223.

17. Milner TA, Shah P, Pierce JP (2000) beta-adrenergic receptors primarily are located on the dendrites of granule cells and interneurons but also are found on astrocytes and a few presynaptic profiles in the rat dentate gyrus. Synapse 36:178-193.

18. Harley CW (2007) Norepinephrine and the dentate gyrus. Prog Brain Res 163:299-318.

19. Lv J, Feng H, Chen L, Wang WY, Yue XL, Jin QH (2017) Activation of $\beta$-adrenoceptor facilitates active avoidance learning through enhancement of glutamate levels in the hippocampal dentate gyrus. Neuroreport 28:973-979.

20. Hansen N, Manahan-Vaughan D (2015) Hippocampal longterm potentiation that is elicited by perforant path stimulation or that occurs in conjunction with spatial learning is tightly controlled by beta-adrenoreceptors and the locus coeruleus. Hippocampus 25:1285-1298.

21. Saygin M, Ozguner MF, Onder O, Doguc DK, Ilhan I, Peker Y (2017) The impact of sleep deprivation on hippocampal-mediated learning and memory in rats. Bratisl Lek Listy 118:408416.
22. Austin KB, Bronzino JD, Morgane PJ (1989) Paired-pulse facilitation and inhibition in the dentate gyrus is dependent on behavioral state. Exp Brain Res 77:594-604.

23. Bronzino JD, Kehoe P, Mallinson K, Fortin DA (2001) Increased extracellular release of hippocampal NE is associated with tetanization of the medial perforant pathway in the freely moving adult male rat. Hippocampus 11:423-429.

24. Neugebauer F, Korz V, Frey JU (2009) Modulation of extracellular monoamine transmitter concentrations in the hippocampus after weak and strong tetanization of the perforant path in freely moving rats. Brain Res 1273:29-38.

25. Devauges V, Sara SJ (1991) Memory retrieval enhancement by locus coeruleus stimulation: evidence for mediation by beta-receptors. Behav Brain Res 43:93-97.

26. Sara SJ, Roullet P, Przybyslawski J (1999) Consolidation of memory for odor-reward association: beta-adrenergic receptor involvement in the late phase. Learn Mem 6:88-96.

27. Straube T, Frey JU (2003) Involvement of beta-adrenergic receptors in protein synthesis-dependent late long-term potentiation (LTP) in the dentate gyrus of freely moving rats: the critical role of the LTP induction strength. Neuroscience 119:473-479.

28. O'Dell TJ, Connor SA, Guglietta R, Nguyen PV (2015) $\beta$-Adrenergic receptor signaling and modulation of longterm potentiation in the mammalian hippocampus. Learn Mem 22:461-471.

29. Ji JZ, Zhang XH, Li BM (2003) Deficient spatial memory induced by blockade of beta-adrenoceptors in the hippocampal CA1 region. Behav Neurosci 117:1378-1384.

30. Stanton PK, Mody I, Heinemann U (1989) A role for Nmethyl-D-aspartate receptors in norepinephrine-induced long-lasting potentiation in the dentate gyrus. Exp Brain 77:517-530.

31. Gray R, Johnston D (1987) Noradrenaline and beta-adrenoceptor agonists increase activity of voltage-dependent calcium channels in hippocampal neurons. Nature 327:620-622.

32. Lee I, Kesner RP (2002) Differential contribution of NMDA receptors in hippocampal subregions to spatial working memory. Nat Neurosci 5:162-168.

33. Li G, Lv J, Wang J, Wan P, Li Y, Jiang H, Jin Q (2016) GABAB receptors in the hippocampal dentate gyrus are involved in spatial learning and memory impairment in a rat model of vascular dementia. Brain Res Bull 124:190-197.

34. Diering GH, Huganir RL (2018) The AMPA receptor code of synaptic plasticity. Neuron 100:314-329.

35. Joiner ML, Lisé MF, Yuen EY, Kam AY, Zhang M, Hall DD, Malik ZA, Qian H, Chen Y, Ulrich JD, Burette AC, Weinberg 
RJ, Law PY, El-Husseini A, Yan Z, Hell JW (2010) Assembly of a beta2-adrenergic receptor--GluR1 signalling complex for localized cAMP signalling. EMBO J 29:482-495.

36. Sanderson JL, Gorski JA, Dell'Acqua ML (2016) NMDA receptor-dependent LTD requires transient synaptic incorpo- ration of $\mathrm{Ca}^{2+}$-permeable AMPARs mediated by AKAP150anchored PKA and calcineurin. Neuron 89:1000-1015.

37. Granger AJ, Shi Y, Lu W, Cerpas M, Nicoll RA (2013) LTP requires a reserve pool of glutamate receptors independent of subunit type. Nature 493:495-500. 